\title{
La conservación del patrimonio cultural en el INAH, México: la labor de la CNCPC a un año de su reestructuración
}

\author{
CNCPC-INAH
}

ste 2014, el Instituto Nacional de Antropología e Historia (INAH) cumple - 75 años, trayecto durante el cual se ha convertido en una de las princi- pales instituciones académicas y científicas no sólo de México sino del mundo en el campo del patrimonio cultural —arqueológico, antropológico, histórico y paleontológico-; legado que el INAH, en virtud de que en eso radica su propósito, investiga, conserva y difunde para fortalecer la identidad y memoria de la sociedad (CUEUM 1972; INAH 2014). Si bien resulta claro que la necesidad de conservar este tipo de bienes nacionales existe desde los inicios del instituto, fue en 1961 cuando creó el Departamento de Catálogo y Restauración del Patrimonio Artístico (DCRPA), que ha derivado en la actual Coordinación Nacional de Conservación del Patrimonio Cultural (CNCPC), de cuyas funciones - a poco más de un año de haber iniciado un proceso de reestructuración fruto de un diagnóstico dirigido a optimizar su desempeñoel presente texto hace una SEMBLANZA.

\section{Orígenes de la CNCPC}

Acerca de la historia de la CNCPC-INAH, descrita parcialmente en algunos documentos (Olivé Negrete y Urteaga Castro-Pozo 1988; Cruz Lara y Magar 1999; Medina-González 2004; Alonso Olvera y García Vierna 2005), baste aquí señalar algunos hitos. Su fundación, que, como ya se mencionó, tuvo lugar en los inicios de la década de 1960, estuvo motivada principalmente por la necesidad de restaurar los murales del ex convento de Culhuacán, en la ciudad de México, donde la DCRPA tenía su sede (Olive y Urteaga 1988:162), tras lo cual se sucedieron varios proyectos de restauración institucional en diversos bienes culturales (Olivé Negrete y Urteaga Castro-Pozo 1988:162). Poco tiempo después, la discusión sobre las alternativas de conservación de los murales de Bonampak, en el estado de Chiapas, México, — descubiertos en 1946, de máxima importancia para la arqueología mesoamericana- concentró en el país reconocidos especialistas internacionales en conservación (Alonso Olvera y García Vierna 2005:53-54). En ese contexto, Manuel del Castillo Negrete promovió, en principio con ayuda de la Organización de las Naciones Unidas para la Educación, la Ciencia y la Cultura (UNESCO, por sus siglas en inglés) 
y, después, de la Organización de los Estados Americanos (OEA), que México fundara un centro de formación y desarrollo en materia de conservación-restauración para América Latina -a la postre, Escuela Nacional de Conservación, Restauración y Museografía (ENCRyM-INAH), que hoy lleva su nombre (Medina-González 2004:10)—, en tanto que la entonces DCRPA-INAH modificó su enfoque al ampliarlo de lo local a lo nacional (Alonso Olvera y García Vierna 2005:45-47). En 1966, se estableció en el ex convento de san Diego, en Churubusco, donde, con una denominación que cambió conforme evolucionaron sus objetivos para convertirse en la CNCPC-INAH, permanece hasta la fecha.

\section{Mandato de la CNCPC-INAH}

La CNCPC-INAH es la mayor área rectora, normativa y ejecutiva en materia de conservación de bienes muebles e inmuebles por destino de carácter paleontológico, arqueológico e histórico en México, competencia que le da el carácter de organización excepcional en el campo patrimonial de América Latina. Su mandato es planificar, coordinar y vincular programas, proyectos y procesos de conservación y restauración del patrimonio cultural en todo el territorio mexicano (CNCPC-INAH 2014a), para lo cual cuenta con una política precisa que al interior del INAH se vincula con diversas dependencias pares, como las coordinaciones de Arqueología, de Monumentos Históricos y de Centros INAH, así como con organismos de injerencia sustancial en la materia, tales como la Dirección de Patrimonio Mundial (DPM), INAH, mientras que al exterior mantiene un trabajo coordinado con el Instituto Nacional de Bellas Artes (INBA), el Consejo Nacional para la Cultura y las Artes (Conaculta), los institutos de cultura de los estados y otras instancias federales responsables de la conservación del patrimonio cultural y/o natural, así como con organismos públicos y privados coadyuvantes en su preservación. Dicha política permite planificar y desarrollar acciones debidamente concertadas en el país, aprovechando los recursos disponibles en diversos nichos de financiamiento y con el apoyo de autoridades de los tres niveles de gobierno y la sociedad civil organizada.

El plan de trabajo de la CNCPC-INAH (2014a) establece nuevas estrategias (Figura 1). Éstas se articulan mediante líneas de acción específicas - de las que se hará un resumen en el siguiente apartado- que, generadas por áreas de actividad, llevan a su concreción diversos equipos interdisciplinarios.

\section{Líneas de acción}

Las áreas de actividad de la CNCPC-INAH se dividen en siete grandes líneas de acción que hacen posible una eficiente planificación de las actividades y los programas a corto, mediano y largo plazos (Figura 2).

\section{Estrategias de la CNCPC-INAH (2013)}

1. Garantizar la conservación integral del patrimonio cultural.

2. Analizar, definir y difundir los lineamientos y criterios que rigen la conservación del patrimonio cultural.

3. Desarrollar y difundir metodologías consistentes para la conservación del patrimonio cultural.

4. Mejorar los conocimientos teóricos, científicos y técnicos de la conservación.

5. Promover la cooperación y el intercambio de conocimientos y experiencias entre profesionales de la conservación, a nivel nacional e internacional.

6. Difundir y socializar los valores atribuidos al patrimonio cultural nacional.

7. Sensibilizar e informar al público, a instituciones relacionadas con el patrimonio cultural y a autoridades en diferentes niveles de gobierno sobre aspectos relativos a la conservación y a su uso racional, de modo que no se afecten su conservación, ni sus valores asociados.

8. Promover la sustentabilidad de la conservación.

9. Definir y proponer indicadores de conservación cuantitativos y cualitativos.

FIGURA 1. Estrategias de la CNCPC-INAH, 2013 (Fuente: CNCPC-INAH 2014).

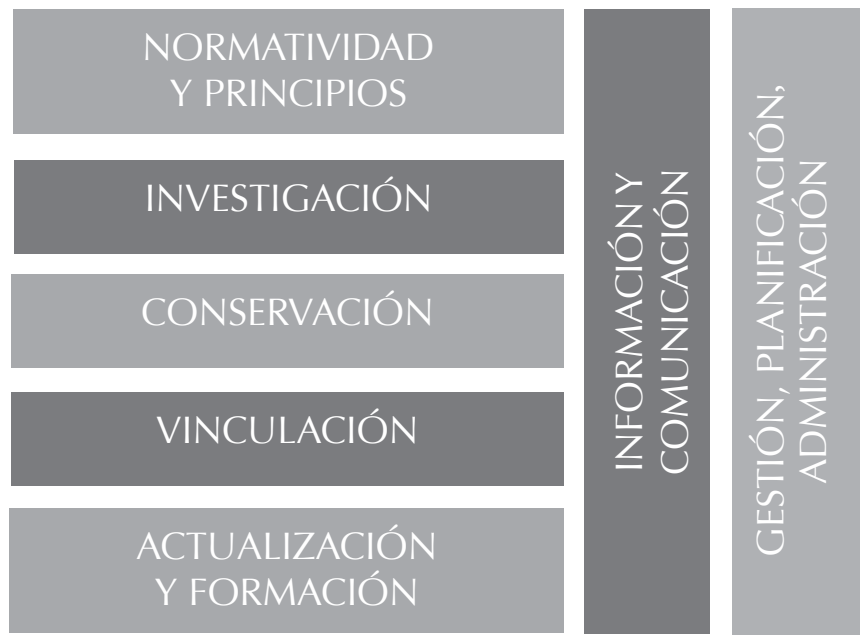

FIGURA 2. Líneas de acción de la CNCPC-INAH, 2013 (Fuente: CNCPCINAH 2014) 
Por medio de esta forma de organización, además de que se garantiza que las actividades respondan a necesidades y prioridades reales, las estrategias aprovechan la sinergia producida entre ellas para potenciar los resultados. Este arreglo se aprecia en los objetivos y actuaciones de cada línea de acción, como se describe a continuación.

\section{Normatividad y principios}

La de normatividad y principios es una de las líneas de acción de la CNCPC-INAH, cuya finalidad consiste en dotar a la institución de un cuerpo de documentos normativos de observancia general para la conservación del patrimonio cultural competencia del INAH, rubro en el que se han desarrollado: una política institucional de conservación, los principios básicos que rigen toda intervención (Figura 3) e, incluso, un glosario de términos para facilitar la comunicación entre profesionales.

Al mismo tiempo, como parte de esta línea de acción, la CNCPC-INAH ha organizado, por un lado, dos seminarios de particular relevancia, uno acerca de la teoría de la conservación y el segundo - en colaboración con la Dirección de Estudios Históricos (DEH) del INAH— sobre normatividad y política pública para la conservación y gestión del patrimonio cultural; y, por el otro, ha impulsado el funcionamiento del Consejo de Conservación de Bienes Muebles (CCBM) y participado de manera regular en los consejos de Arqueología y de Monumentos Históricos, todos ellos, instancias consultivas de la Di- rección General (DG) del INAH. Como corolario de estas actividades, se han revisado y actualizado tanto el manual de organización interno de la CNCPC-INAH como los procedimientos para autorizar proyectos de intervención en conservación propuestos ya sea por restauradores del propio instituto o por profesionales privados.

\section{Investigación}

En esta línea de acción, la CNCPC-INAH efectúa labores de investigación aplicada que responden a los requerimientos y demandas planteados en los diferentes proyectos de conservación del patrimonio cultural que ahí se ejecutan. Adicionalmente se ha programado una agenda de investigación institucional, de la que se desprenden los futuros proyectos, que consta de los siguientes rubros: a) caracterización y diagnóstico de materiales culturales (Figura 4); b) y c) evaluación y desarrollo, respectivamente, de productos y métodos de intervención; $d$ ) monitoreo de tratamientos de conservación; e) investigación y recuperación de la historia de algunas comunidades que trabajan en la preservación de su patrimonio, y f) y g) investigación en conservación para el diseño de indicadores, y sobre su teoría y normatividad, respectivamente.

\section{Conservación}

Aunque la CNCPC-INAH tiene un mandato amplio en materia de conservación, su desempeño fundamental ha sido como instancia ejecutora de proyectos y, de forma más

\section{SÍNTESIS DE PRINCIPIOS NORMATIVOS DE CONSERVACIÓN PLANTEADOS}

POR LA CNCPC-INAH

Para que una acción de conservación sea legítima, ha de regirse por los siguientes principios:*

- Debe respetar la integridad del patrimonio cultural basándose en la comprensión y consideración tanto de su materia, factura, aspecto o imagen, valores, significados, usos, asociaciones y contexto como de los actores sociales vinculados con él.

- Debe realizarse mediante un proceso metodológico fundado en un trabajo interdisciplinario con la finalidad de que éste contribuya al estudio, comprensión y transmisión de los valores del patrimonio cultural.

- Las decisiones que se tomen respecto de la conservación deben recurrir a la experiencia, los conocimientos, los juicios y la pericia de profesionales especializados en la materia.

- Debe dar preferencia a las acciones preventivas sobre las acciones directas.

- Debe documentarse, procurando que sus resultados se socialicen o publiquen.

- Deberá buscar alcanzar la más alta calidad posible, teniendo en cuenta la responsabilidad social y profesional que comporta la conservación del patrimonio cultural, la cual se considera una labor de interés público.

* La aplicación e interpretación de estos principios debe ser integral, pues son complementarios. La conservación del patrimonio cultural es de interés público y conlleva responsabilidades.

FIGURA 3. Principios de conservación en elaboración por la CNCPC-INAH (Cortesía: CNCPC-INAH 2014). 


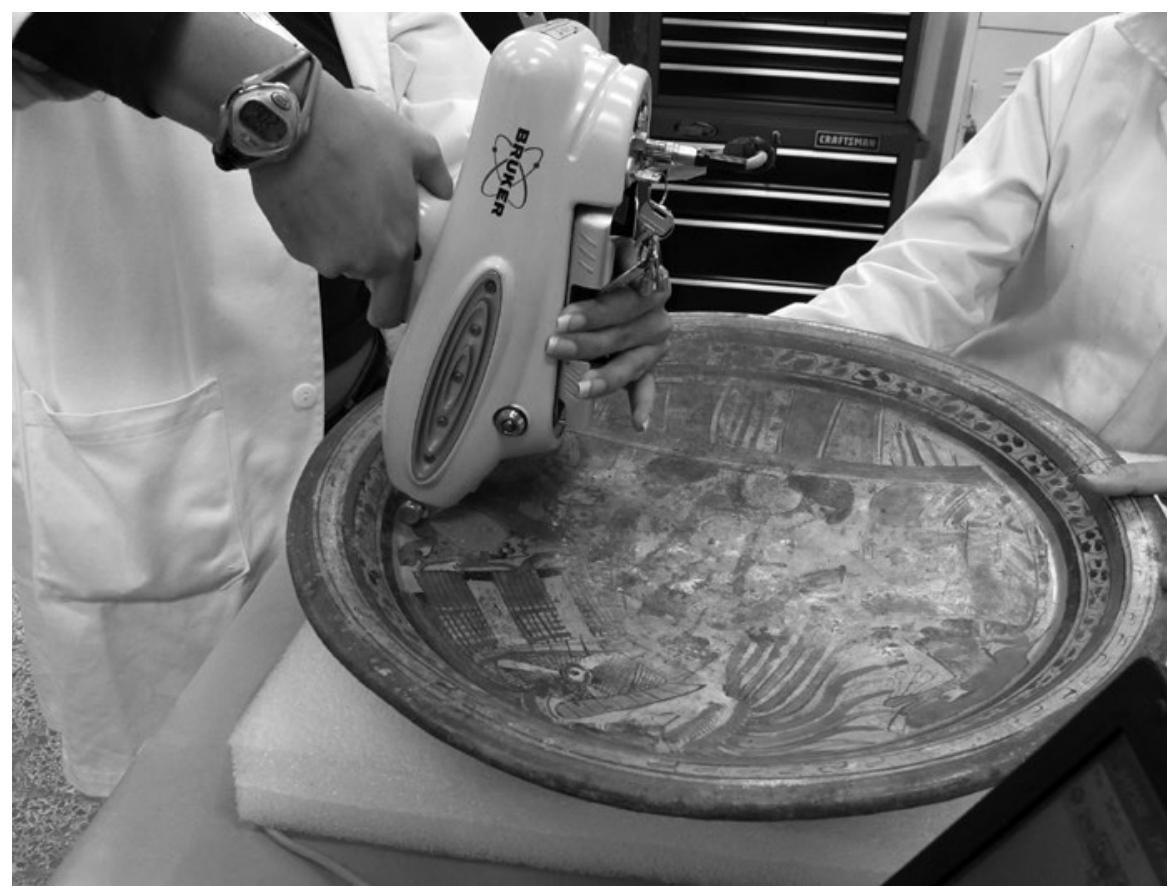

FIGURA 4. Análisis elemental por espectrometría de fluorescencia de RX en una cerámica maya (Fotografía: Carmen Noval 2013; cortesía: CNCPC-INAH ).

limitada, como instancia rectora y normativa. Por esta razón, desde el 2013 se ha procurado que la coordinación asuma una cabal comprensión de los problemas en conservación en el país para lograr una mayor cobertura en la atención del patrimonio mexicano. Para esto se han planteado seis programas nacionales, que se desarrollarán progresivamente y a largo plazo, que atienden aproximaciones (por ejemplo, conservación preventiva o para grupos sociales) o temáticas (por mencionar sólo dos: para patrimonio arqueológico o para acervos documentales). Asimismo, la CNCPC-INAH ejecuta proyectos específicos y actividades generales en una diversidad de bienes culturales, ya sea in situ o en sus laboratorios (material arqueológico, pintura de caballete, documentos gráficos, escultura policromada, textiles, entre otros), cuyo impulso, si bien independiente, repercute en el desarrollo de cada uno de los seis programas (Figura 5). También considera solicitudes externas o situaciones de emergencia y realiza asesorías, dictámenes, evaluaciones, supervisiones y peritajes relacionados con proyectos del instituto $u$ otras instancias públicas y privadas.

\section{Vinculación}

Esta línea de acción, mediante la que se establecen a escala nacional canales de comunicación permanente con los restauradores del INAH y los directivos de las diversas instituciones del ramo, tiene como cometido concretar vínculos con instancias externas al INAH que promueven o llevan a cabo acciones de conservación. Este esquema de comunicación e intercambio busca definir las necesidades de apoyo de los restauradores, atender requerimientos vía las áreas pertinentes, así como difundir y socializar la normatividad y los procedimientos vigentes en materia de conservación para instrumentar su correcta aplicación a través de una capacitación directa.

Finalmente, en el 2013, la CNCPCINAH promovió una reunión nacional de restauradores del INAH que hizo posible elaborar un análisis conjunto sobre el funcionamiento de las áreas de conservación en nuestro instituto (Figura 6), a partir de la cual se ha convocado a reuniones regionales para examinar problemáticas específicas de cada territorio, útiles, asimismo, para establecer redes de colaboración regionales. Como estos encuentros han rendido frutos, se pretende que aquella reunión con restauradores de todo el país auspiciada por la CNCPC-INAH se formalice como una actividad anual, ya que con ello se dispondrá de un foro importante de intercambio en nuestro ámbito profesional.

\section{Actualización y formación}

Con esta línea de acción, en su totalidad referida tanto al país como al extranjero, lo que se propone es consolidar las labores de actualización y formación de los restauradores del INAH, así como apoyar las tareas académicas, de reflexión y educación en torno de la conservación. De esta manera, con la finalidad de estrechar la relación de los distintos agentes que se vinculan y usan el patrimonio, se promueve el análisis e intercambio en dos vías: por un lado, mediante la difusión de las labores y los estudios que se llevan a cabo -insistimos, en cualquier lugar del mundo- $y$, por el otro, por medio de la organización de cursos, talleres y foros de discusión con especialistas. Para alcanzar esta meta, permanentemente se proponen convenios y acuerdos con escuelas y centros de investigación y de trabajo, entre los que han destacado los seminarios en materia de conservación de piedra y papel realizados en el marco de la iniciativa de colaboración con el Programa para la Conservación del Patrimonio Cultural en América Latina y el Caribe (LATAM) del International Centre for the Study of the Preservation and Restoration of Cultural Property (ICCROM) (Figura 7).

\section{Información y comunicación}

Como la difusión del trabajo realizado por la CNCPC-INAH es fundamental, tanto en materia de rendición de cuentas 
como en la de la transparencia que se debe a la ciudadanía para hacerla partícipe del patrimonio que le pertenece (Figura 8), en distintos medios se divulga información veraz, precisa y de buena calidad sobre la conservación. En esta línea, su página web (CNCPC-INAH 2014b) constituye un mecanismo fundamental.

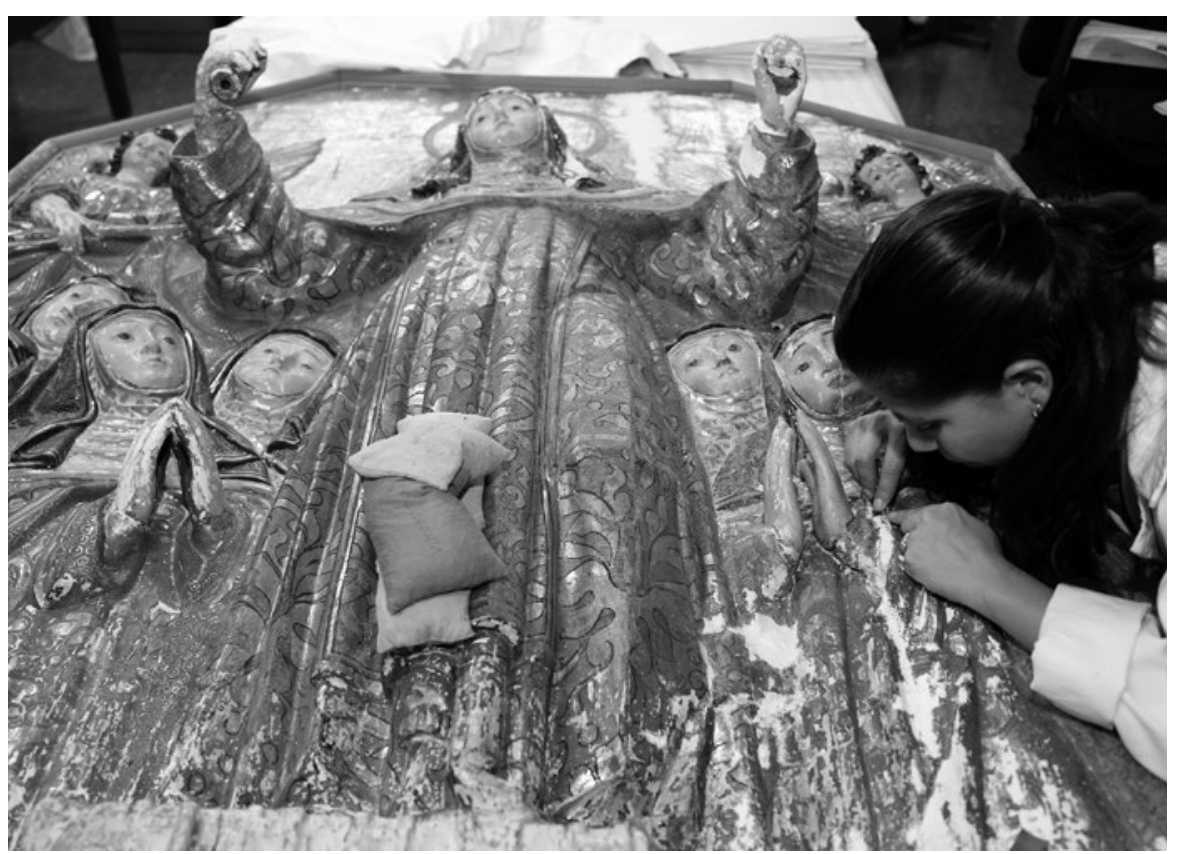

FIGURA 5. Restauración de la tabla de santa Clara, proveniente de Atlixco, Puebla, en el laboratorio-taller de restauración de escultura policromada de la CNCPC, 2013 (Fotografía: Carmen Noval 2013; cortesía: CNCPC-INAH).

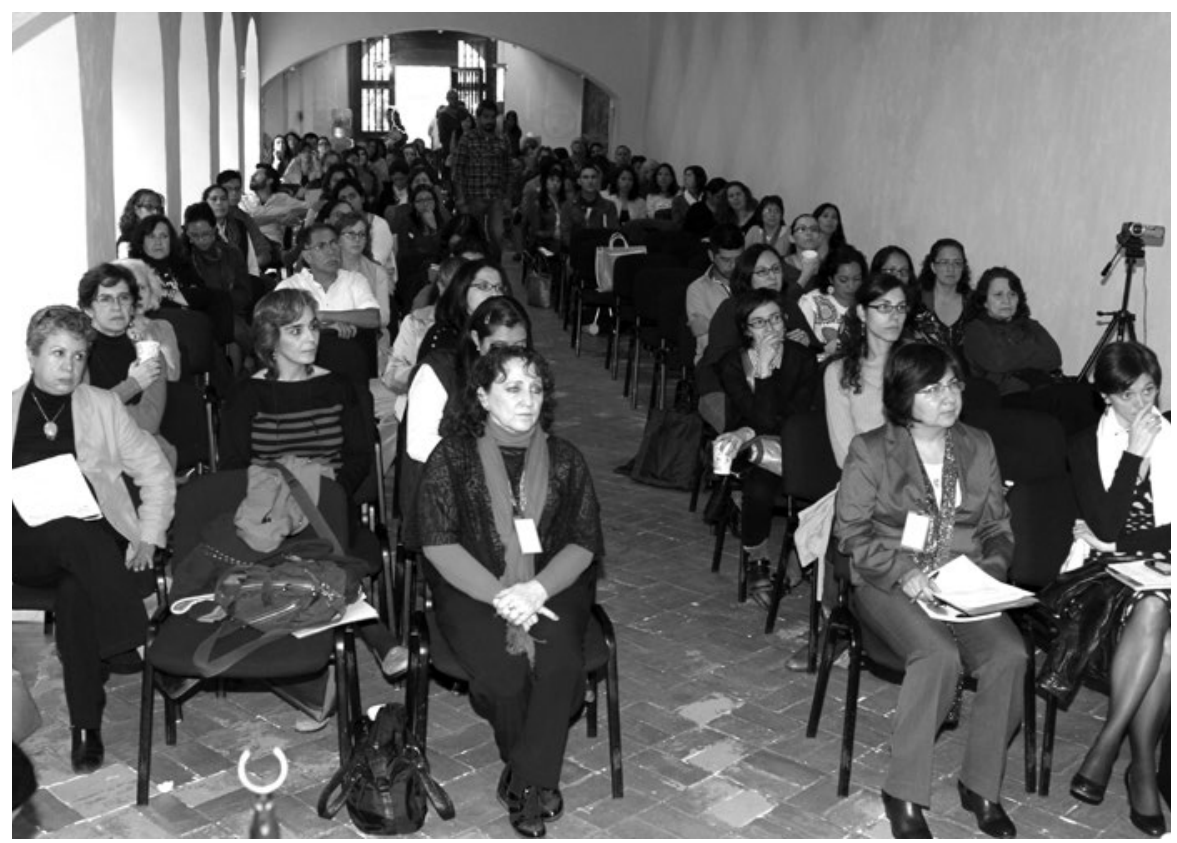

FIGURA 6. Reunión nacional de restauradores del INAH en el Museo Nacional de las Intervenciones, octubre de 2013 (Fotografía: Carmen Noval 2014; cortesía: CNCPC-INAH).
De conformidad con el mandato de la CNCPC-INAH, desde su creación se han incrementado tanto sus funciones como sus actividades. Respecto de estas últimas, las planteadas durante su reestructuración tienen el objeto de fortalecer una de las coordinaciones sustantivas del instituto. Actualmente se trabaja con el propósito de que el organigrama responda a las necesidades y realidades contemporáneas.

\section{A manera de una breve conclusión}

El proyecto actual de la CNCPC-INAH pretende perfeccionar el marco normativo en la materia y consolidar una política de conservación, acciones que, en conjunto, permitirán definir estrategias claras para que el instituto asegure la conservación del patrimonio cultural conforme a los retos y desafíos de la actualidad y, con ello, efectivamente, robustecer las operaciones de una organización excepcional en ese ámbito en América Latina. Su página web oficial es: [http://www.conservacionyrestauracion.inah.gob.mx].

\section{Referencias}

Alonso Olvera, Alejandra y Valeria A. García Vierna

2005 “Propuesta de lineamientos teóricos y prácticos de la Subdirección de Conservación Arqueológica de la Coordinación Nacional de Conservaciones del Instituto Nacional de Antropología e Historia", en Diana Magaloni (ed.), Lineamientos y limitaciones en la conservación. Pasado y futuro del patrimonio. $10^{\circ}$ Coloquio del Seminario de Estudio y Conservación del Patrimonio Cultural, México, IIES-UNAM: 43-84.

CNCPC-INAH

2014a "Plan de trabajo CNCPC-INAH 2014", documento electrónico disponible en [http://www.mener.inah. gob.mx/archivos/Plan_Trabajo_CNCPC_2014.pdf], consultado en junio del 2014.

2014b Coordinación Nacional de Conservación del Patrimonio Cultural, 


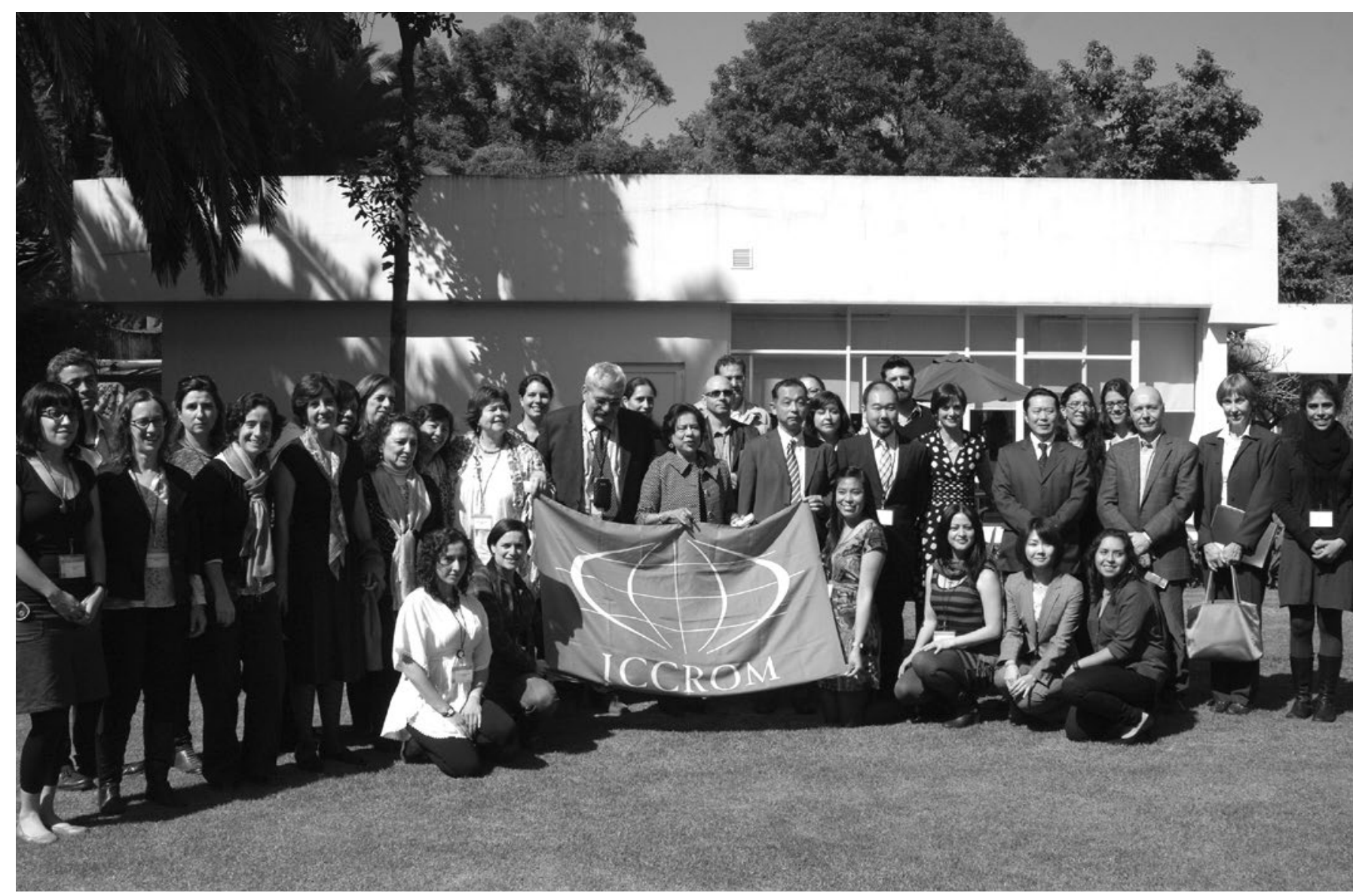

FIGURA 7. Participantes de los seminarios LATAM-ICCROM en conservación de papel y piedra organizados por la CNCPC en noviembre de 2013 (Fotografía: Carmen Noval 2013; cortesía: CNCPC-INAH).

documento electrónico [página web] disponible en [http:// www.conservacionyrestauracion inah.gob.mx], consultado en junio del 2014.

Cruz Lara, Adriana y Valerie Magar.

1999 "Conservation in Mexico", en Janet Bridgland (ed.), Proceedings of $12^{\text {th }}$ Triennial Meeting Lyon, 29 August- 3 September, Londres, ICOM/James \& James: 177-182.

\section{CUEUM}

1972 "Ley Federal de Monumentos y Zonas Arqueológicos, Artísticos e Históricos", Diario Oficial de la Federación, México, Congreso de la Unión de los Estados Unidos Mexicanos (CUEUM).

INAH

2014 Quiénes somos, documento electrónico disponible en [http://www.inah.gob.mx/iquienes-somos], consultado en julio de 2014.

Medina-González, Isabel

2004 "Conservación arqueológica en México: una historia en el contexto internacional", ponencia presentada en el Seminario Permanente de Conservación Arqueológica, México, CNCPC-INAH: 10.

Olivé Negrete, Julio César y Augusto Urteaga Castro-Pozo (coords.)

1988 INAH. Una historia, México, INAH.

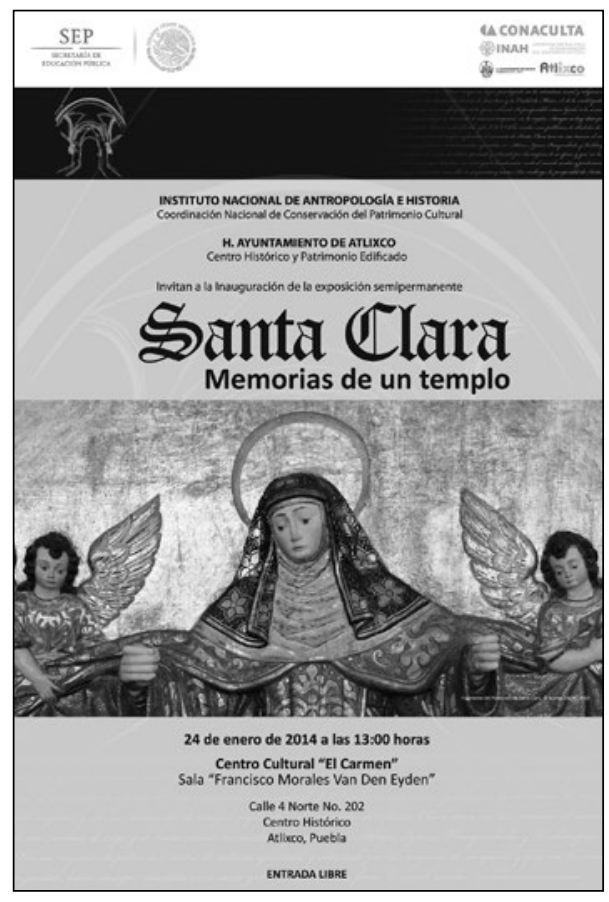

FIGURA 8. Cartel de la exposición organizada en AtlixCo, Puebla, con motivo de la entrega de obras restauradas (Fotografía: Carmen Noval 2014; cortesía: CNCPC-INAH). 


\section{Resumen}

En este texto se presenta una SEMBLANZA de las funciones de la Coordinación Nacional de Conservación del Patrimonio Cultural (CNCPC), Instituto Nacional de Antropología e Historia (INAH), México, a poco más de un año de haberse iniciado un proceso de reestructuración, fruto de un diagnóstico para optimizar su desempeño. Se esbozan los orígenes de la CNCPC-INAH y su mandato, a la vez que se hace una breve descripción de sus líneas estratégicas con el fin de ilustrar las acciones de una organización excepcional en el ámbito del patrimonio cultural latinoamericano.

\section{Palabras clave}

CNCPC-INAH; conservación; patrimonio cultural; México; líneas estratégicas

\section{Abstract}

This text provides an OVERVIEW of the functions of the Coordinación Nacional de Conservación del Patrimonio Cultural (National Coordination of the Conservation of Cultural Heritage, CNCPC), Instituto Nacional de Antropología e Historia (National Institute of Anthropology and History, INAH), Mexico, describing the results of little more than a year of reorganization processes, initiated in order to optimize the performance of the CNCPC-INAH. An outline of origins and mandate of the CNCPC-INAH as well as a brief description of the organization's strategic directions, illustrate the activities of an exceptional institution in the Latin-American field of cultural heritage.

\section{Key words}

CNCPC-INAH; conservation; cultural heritage; Mexico; strategic directions

Título en Inglés: The Conservation of Cultural Heritage at INAH, Mexico:

the Activities of CNCPC after a year of Structural Reorganization

Postulado/Submitted 07.07.2014

Aceptado/Accepted 18.07.2014 